\title{
Research of the Auditing System and Legal Control of Electronic Exchanges
}

\author{
Ming Chen \\ School of Business, Soochow University, Suzhou, P.R. China \\ cm192@163.com
}

Keywords: Electronic exchanges; Auditing; Legal control; Financial risk; Information disclosure mechanism

\begin{abstract}
Many electronic exchanges of raw materials, artworks, precious metals and agricultural products have been established in China in recent years. Because of the auditing and legal control failed to follow-up timely, they appear a variety of problems in auditing, legal control and operation process. To deal with their financial risks well and fight against all risks of bankruptcy, illegal and criminal related to the electronic exchanges, we must strengthen the construction of auditing and legal control; establish the system of auditing and financial safety regulation; improve the information disclosure mechanism of the electronic exchanges; establish and perfect the internal and external audit system of electronic exchange; establish the classification system of electronic exchanges, conduct auditing and regulation to the electronic exchanges by classification; amend relevant laws and regulations, regulate mandatory audit system; and combat all kinds of illegal activities which involving electronic transactions.
\end{abstract}

\section{电子化交易所的审计制度与法律规制研究}

\author{
陈铭 \\ 苏州大学 商学院，中国 江苏 苏州 215002 \\ cm192@163.com
}

摘要: 我国近年来相继建立了大宗原材料、贵金属、艺术品、农产品等各类电子化交易所, 其在现代经济体系中的定位与作用，可用新兴古典经济学模型进行分析。但由于其审计制度 与规制制度未能及时跟进，在实际运作过程中出现了各种问题。为减少其可能面临的巨大财 务风险，避免其破产、违法及犯罪的可能，必须加强电子化交易所的审计制度及法律规制建 设，建立完善的审计制度及金融安全规制。并应健全其信息披露机制，设立金融安全性法规， 建立健全电子化交易所内部与外部审计制度; 建立电子化交易所分级制, 分级分类进行审计 与规制; 修改相关法规，规范强制审计制度，打击涉及电子化交易的各类违法行为。

关键词：电子化交易所；审计制度；法律规制；金融风险；信息披露机制

\section{1. 引言}

中国近年来各类电子化交易所如雨后春笋般地建立起来。据估计，除国家正式批准的股票交 易所与商品期货交易所，以及中国金融期货交易所之外，全国尚有各类产权交易场所 300 多 家，交易量将超过万亿元。这些电子化交易所良莠不齐，除少部分运行比较规范外，大多数 没有得到有效管理, 审计制度与法律规制严重不全, 出现秩序混乱、风险严重、甚至欺诈投 资者等现象，严重扰乱了正常经济秩序，形成价格的非理性大起大落。

关于基于电子交易的各类产权交易所 [1] [2] 审计制度 [3] [4] [5] 与法律规制 [6] [7] 问题的研 究已有不少。特别是，自国务院发布《关于清理整顿各类交易场所切实防范金融风险的决定》 [8] 以来，我国正式将清理整顿从事产权交易的各类电子化交易场所提上了议事日程。但交易 
所清理工作既取得了一定的成效 [9], 也在一定程度上受到了相关方面的抵制, 很多原来的交 易所纷纷改头换面, 卷土重来, 亟待引起重视 [10]。对这些电子化交易所进行有效审计与法 律规制, 成为一个亟待解决的问题, 本文拟对此作一研究。

\section{2. 电子化交易所分类与现状}

我国电子化交易所发展较快, 从交易品种分，主要有以下几类:

2.1. 大宗原材料电子化交易场所

具体包括: 石化、煤炭、有色金属等，如天津渤海商品交易所、河北金江不锈钢交易所、山 西中太煤炭电子交易市场、天津天保大宗煤炭交易市场、天津稀有金属交易所、浙江舟山大 宗商品交易中心等。这些大宗原材料交易所有些规模较大、运作比较规范, 有较好的信誉, 有些成立时间较短、盲目上马, 出现了各种问题。

2.2. 贵金属电子化交易场所

主要指黄金、白银等贵金属的电子化交易，如湖南维财大宗贵金属交易所等，这类交易所往 往采用保证金交易, 由于黄金、白银的国际价格变化幅度很大，导致投资者很容易爆仓，血 本无归。

2.3. 艺术品电子化交易场所

著名的有天津文化艺术品交易所、山东泰山文化艺术品交易所等, 由于文化艺术品的独一无 二性, 产品很难复制, 价值很难估计, 市场变现能力低, 其价格存在上升、下降的很大空间, 因此，这类产品交易的炒作性往往很强，价格大起大落，套牢了很多投资者。

2.4. 农产品电子化交易场所

很多该类电子化交易场所出现在大型农产品集散地, 交易品种从大蒜、生姜、各类蔬菜、牲 畜无所不包, 如山东寿光蔬菜交易所, 天津国盛农产品交易所等。近年来, 大量的农产品电 子交易所在各地成立, 且很多运作很不规范。

2.5. 其它电子化交易所

其它还有包括进行碳排放、固体废弃物、金融资产、药品等交易的电子化交易所。总体而言, 各种门类的电子化产权交易所都在逐渐出现, 似乎只要是有价格的东西, 都可以拿来交易。

\section{3. 电子化交易所的作用与经济学定位}

从电子化交易所的作用与经济学定位来看, 电子化交易场所在组织交易, 实现交易市场整合 方面起着积极作用。对其在现代经济体系中的定位, 可用新兴古典微观经济学的专业化与分 工理论模型进行分析:

对于专门从事交易整合的电子化交易所而言，其所生产的产品就是 “交易整合” 本身。交易 整合本身作为产品是每个市场参与者都需要的, 因此具有较高的偏好参数, 并且可以认为交 易整合也是专业化程度较高的产品。可以证明, 把 “交易整合” 本身作为贸易产品（即购买 和出售 “交易整合”服务，以及存在电子化交易所）可以得到较高的人均收入水平:

为方便起见, 假设生产一消费体系中只有两种产品 $X, Y$, 其中 $X$ 为一般产品, $Y$ 为 “交易整 合” 产品。

在新兴古典经济学框架中 $[11]$ : 组态 $(X / Y)$ 和 $(Y / X)$ 的效用可以表示为:

$\ln u(x / y)=\alpha\left(2 \ln x+\ln r-\ln M_{x y}\right)+\gamma \ln z+\alpha \ln K$

$\ln u(y / x)=\alpha\left(2 \ln x+\ln r+\ln M_{x y}\right)+\gamma \ln z+\alpha \ln K$

式中 $x=[2 \alpha /(3 \alpha+\gamma)]^{\alpha} / 2, z=[\gamma /(3 \alpha+\gamma)]^{\alpha}, \quad r=[\alpha /(3 \alpha+\gamma)]^{\alpha}$,

效用均等条件为: $E=\ln u(y / x)-\ln u(x / y)=2 \alpha \ln m_{x y}=0$

设 $\mathrm{u} *$ 为真实收入, $\mathrm{M} *_{\mathrm{xy}}$ 为该角点均衡中选择不同组态的相对个数, 由 (1)、(2)、(3) 式可得: 
$d M_{x y}^{*} /\left.d \alpha\right|_{\alpha=\gamma}=-(\partial E / \partial \alpha) /\left.\left(\partial E / \partial M_{x y}\right)\right|_{\alpha=\gamma}=0$

式中 $\partial E /\left.\partial \alpha\right|_{\alpha=\gamma}=2 \ln M_{x y}=0$, 因为如果 $\alpha=\gamma$, 则 $M_{x y}=1$; 同时可得：

$\partial \ln u * /\left.\partial \alpha\right|_{\alpha=\gamma}>0$

因此有: $d \ln u * /\left.\partial \alpha\right|_{\alpha=\gamma}=\partial \ln u * /\left.\partial \alpha\right|_{\alpha=\gamma}+\left.\left(\partial \ln u * / \partial M_{x y}\right)\left(d M_{x y}^{*} / d \alpha\right)\right|_{\alpha=\gamma}>0$

即: 以偏好参数较大的产品作为贸易商品的角点均衡得出较高的人均收入水平。再假设 $\alpha=$ $\beta=\gamma, a=b=h$ 。若:

$x=[2 a /(3 a+c)]^{a} / 2, z=[c /(3 a+c)]^{a}, M_{x y}=[2 a /(3 a+c)]^{a}$,

分别用 $[2 a /(3 a+c)]^{a} / 2 、[c /(3 a+c)]^{a} 、[2 a /(3 a+c)]^{a}$ 代换（1）、(2) 式中的 $\mathrm{X} 、 \mathrm{z} 、 \mathrm{M}_{\mathrm{xy}}$, 即得:

$\partial \ln u * /\left.\partial a\right|_{a=c}>0$

$d M_{x y}^{*} /\left.d a\right|_{a=c}=-(\partial E / \partial a) /\left.\left(\partial E / \partial M_{x y}\right)\right|_{a=c}=0$

式中 $\partial E /\left.\partial a\right|_{a=c}=0, \mathrm{E}$ 由 (3) 式给出。由此得:

$d \ln u * /\left.\partial \alpha\right|_{a=c}=\partial \ln u * /\left.\partial \alpha\right|_{a=c}+\left.\left(\partial \ln u * / \partial M_{x y}\right)\left(d M_{x y}^{*} / d \alpha\right)\right|_{a=c}>0$

即：以专业化报酬较高的产品作为贸易商品的角点均衡得出较高的人均收入水平。

由于上述两个结论的成立, 且在 “交易整合” 是专业化与偏好参数都较高的产品的条件下, 有独立的交易整合商存在得到较高的人均收入水平。电子化交易所即是 “交易整合” 这一产 品的提供者。其存在创造了较高的人均收入水平, 即由原来大宗原材料、贵金属、艺术品、 农产品等交易的双边市场转变为中心市场，电子化交易所的运行机制如图 1 所示:

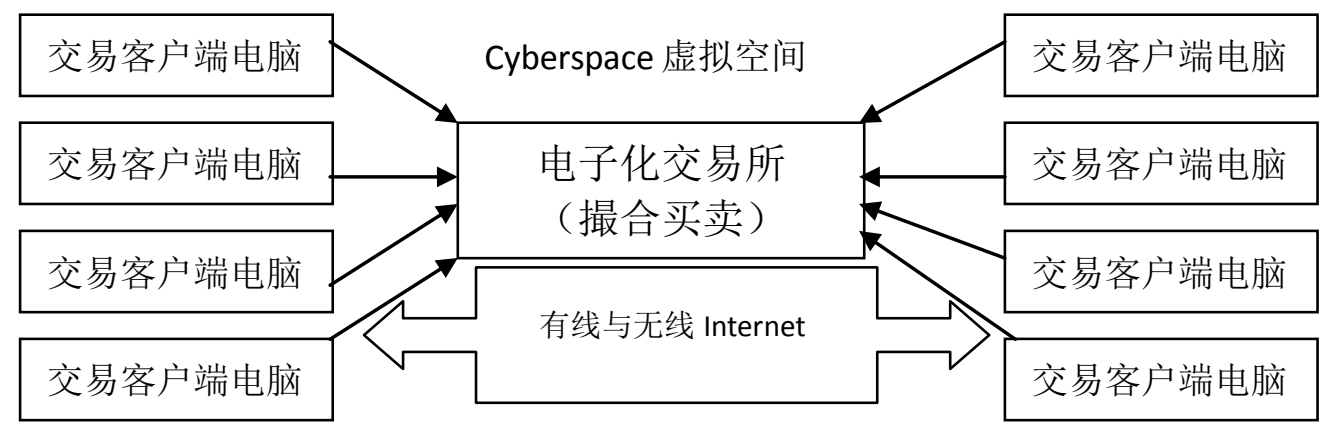

图 1 电子化交易所的运行机制

概而言之, 由于电子技术提高了交易效率, 使得 “交易整合” 这一产品从自给自足转变为分 工。自给自足时, 没有专业化平台的存在, 由于分工, 形成了专业化的电子化交易所。20世 纪 90 年代以来信息技术的飞速发展以及信息化基础设施的普及, 创造了电子化高效率交易这 一条件，使得电子化交易所的出现成为可能以及必须。

\section{4. 电子化交易所的现存审计与规制问题}

电子化交易所由于审计制度及法律规制未能及时跟进，在实际运作过程中出现了各种问题。 主要表现于以下方面:

4. 1. 商品交易成为逐利游戏, 背离商品内在价值

根据价值规律, 商品的价格应围绕其价值上下波动。但在电子化交易所, 由于投资者交易的 目的发生了变化, 其交易的目的不是为了拥有商品本身, 而是为了通过低买高卖, 博取差价。 同时, 由于电子化交易所内聚集了大量资金, 这些资金可以通过不断买入 (卖出), 在一段时 间内不断推升 (打压) 价格, 从而人为地造成 “理性泡沫”。在这种条件下，理性的投资者只 
能跟风操作, 才能盈利, 如果坚持价值判断, 不跟风, 反而会亏得很惨。同时, “理性泡沫” 发展到一定程度, 又会引起 “非理性泡沫” 的产生。吸引大量场外投资者盲目跟风，在高位 买进，一有风吹草动，又同时恐慌，一起卖出，价格急转直下。

4.2. 缺乏审计制度与法律规范, 存在严重价格操纵与利益输送行为

如果说如前所述 “击鼓传花” 游戏尚在合法范围内的话, 由于缺乏必要的审计制度与法律规 范, 在很多电子化交易所内出现的严重价格投机与价格操纵行为却已超出了法律允许的范围。 少数人钻法律空子，利用自己各方面的影响力操纵价格，从中渔利。有些交易所不是向每个 投资者提供公平、透明的交易机制，而是想方设法，通过各种内幕方法，层层设 “巧”，以交 易之名, 行操纵之实。

4. 3. 违背法律规定，实施 “对奢”，欺计投资者

个别电子化交易所实际已存在严重违法现象，表现为其股东直接参与交易，与投资者实施 “对 奢”。在这些交易场所的电子盘中，投资者进行的交易活动表面已经完成，但实际上并未真实 发生，当价格向对投资者不利方向发展时，投资者被迫进行平仓，损失归已，而收益却被交 易所获得; 当价格向对投资者有利方向发展时，交易所通过网络阻断、致使投资者的盈利无 法实现，直至变为亏损。

\section{5. 结论：电子化交易所的审计制度与法律规制建设}

中国各地近年来相继建立的各类电子化交易所一方面活跃了市场交易，为广大商品购买者、 使用者提供了便利, 也带动了当地的社会经济发展, 但同时也存在着以上述及的各种审计与 规制问题，有些问题还相当严重，必须引起高度重视。

笔者认为, 清理整顿各类交易场所, 不是要将此类电子化交易场所全部关闭, 清理整顿的重 点，应是努力建立、健全电子化交易所的相关的审计制度与法律规制，使其在审计制度与法 律规制有效管理下，迅速规范化，特别是在其防范金融风险方面有法可依，从而切实有助其 防范金融风险，建立遵法守规、信誉良好、金融安全、投资者信得过、市场能接受的电子化 交易所。为此，应尽快建立、健全以下电子化交易所的法律规制：

5. 1. 尽快建立、完善电子化交易所设立、变更、注销、登记的法律、法规

第一步是有法可依，近年来很多电子化交易场所存在的问题及其种种表现，往往是因为无法 可依。因此，应尽快制定全国性的关于电子化交易所设立、变更、注销、登记等相关流程的 法律规定, 特别是在其设立时, 为其建立较高的壁垒。法律、法规应详细规定各类电子化交 易所设立时各自应具备的资金与其它条件，应考虑到近年来出现的各类相关问题，特别是与 金融安全相关的问题，从源头上加以预防。

5. 2. 及早制定全国性、法规性的各类电子化交易所的内部审计制度与交易规则

同时，还应及早制定全国性、法规性的各类电子化交易所的内部审计制度交易规则，交易规 则可采用做市商、集中竞价、电子撮合等标准化合约交易方式，或禁止采取匿名交易、并同 时控制权益持有人数量。从而从规则上有效避免各种交易陷阱的产生可能性。

5.3. 设立金融安全性法规，建立健全电子化交易所内部与外部审计制度

防范系统性、区域性金融风险, 确保社会稳定, 是这次国务院清理整顿各类交易场所的重要

目标。为此，应建立健全电子化交易所内部与外部审计制度，专门制定有关电子化交易所金 融安全的法规, 定量、实时监控各类电子化交易所的日常运作。可以考虑实施产权交易场所 日常营运金融实况的逐日报告制，定量、实时监控电子化交易所各类数据。

5. 4. 建立电子化交易所分级制的相关法规、办法, 分级分类进行审计与规制

同时，可考虑建立电子化交易所分级制的相关法规、办法，尽快实施电子化交易所的分级制 管理, 分级分类进行审计与规制。在统一高标准要求的前提下, 具体依照不同的定量标准划 分不同的等级，各类不同的电子化交易所根据自身的特点与条件申请相关等级，由有关部门 根据其具体情况进行核准。这样有利于投资者根据不同级别来选择适合自己的电子化交易所, 以降低投资风险，切实保护投资者利益。 


\section{6. 致谢}

感谢江苏省哲学社会科学基金规划项目（EYB022）的资助。

\section{参考文献}

[1] Co R, Berkowitz J, Patel J, et al. Detection of Potential Abusive Trading Behavior in Electronic Markets, US20150081505[P]. 2015.

[2] Aidov A, Daigler R T. Depth Characteristics for the Electronic Futures Limit Order Book[J]. Journal of Futures Markets, 2015, 35(6):542-560.

[3] Staub, Walter A. INTRODUCTION : Auditing Developments During the Present Century[J]. Journal of Cancer, 2016, 7(74):377-390.

[4] Yong Yu, Liang Xue, Man Ho Au, et al. Cloud data integrity checking with an identity-based auditing mechanism from RSA[J]. Future Generation Computer Systems, 2016.

[5] Cao J, Chen F, Higgs J L. Late for a very important date: financial reporting and audit implications of late 10-K filings[J]. Review of Accounting Studies, 2016:1-39.

[6] Vokshi N B, Xhelili-Krasniqi F, Ujkani S. Evaluation of the Accounting Legal Regulation and its Role in the Quality of Financial Reporting [C]// International Scientific Conference. 2015.

[7] Shi K, Hu D. Internet Financial Innovation and Legal Regulation Selection[J]. Finance \& Economics, 2015:158-161.

[8] 国务院关于清理整顿各类交易场所切实防范金融风险的决定 [G].http://www.chinapolicy.net /bencandy.php?fid=141\&id=7412. 2011-11-24.

[9] 魏书光.大宗商品交易所整顿11月中或见效[N]. 证券时报. 2015-11-6.

[10]全国交易场所清理整顿情况简述[R]. http://wenku.baidu.com/link?url=11-tR9a64QhAbrNKV _bwTUbDE2m1Z6hNky.2015-8-20.

[11]杨小凯,黄有光.专业化与经济组织[M]. 经济科学出版社.2000.pp149-157.

\section{References}

[1] Co R, Berkowitz J, Patel J, et al. Detection of Potential Abusive Trading Behavior in Electronic Markets, US20150081505[P]. 2015.

[2] Aidov A, Daigler R T. Depth Characteristics for the Electronic Futures Limit Order Book[J]. Journal of Futures Markets, 2015, 35(6):542-560.

[3] Staub, Walter A. INTRODUCTION : Auditing Developments During the Present Century[J]. Journal of Cancer, 2016, 7(74):377-390.

[4] Yong Yu, Liang Xue, Man $\mathrm{Ho} \mathrm{Au}$, et al. Cloud data integrity checking with an identity-based auditing mechanism from RSA[J]. Future Generation Computer Systems, 2016.

[5] Cao J, Chen F, Higgs J L. Late for a very important date: financial reporting and audit implications of late 10-K filings[J]. Review of Accounting Studies, 2016:1-39.

[6] Vokshi N B, Xhelili-Krasniqi F, Ujkani S. Evaluation of the Accounting Legal Regulation and its Role in the Quality of Financial Reporting [C]// International Scientific Conference. 2015.

[7] Shi K, Hu D. Internet Financial Innovation and Legal Regulation Selection[J]. Finance \& Economics, 2015:158-161. 
[8] China State Council: The Decision on the Prevention of Financial Risks in the Clean up and Rectify all Types of Trading Venues[G].http://www.chinapolicy.net/bencandy.php?fid= 141\&id=7412. 2011-11-24. (in Chinese)

[9] Wei, Shu-guang. The Rectify of Commodity Exchanges May Bear Fruit in November[N] . Securities Times. 2015-11-6. (in Chinese)

[10] The Brief Situation of the Clean up and Rectify of Trading Place[R]. http://wenku.baidu.com /link?url=11-tR9a64QhAbrNKV_bwTUbDE2m1Z6hNky.2015-8-20. (in Chinese)

[11] Yang,Xiao-kai, Huang, You-guang. Specialization and Economic Organization [M]. Economic Science Press.2000.pp149-157. (in Chinese)

作者简介: 陈铭 (1968-) , 男, 江苏省苏州市人, 同济大学经济管理学院博士后, 苏州大 学商学院副教授，主要研究方向为电子商务、审计, E-mail：cm192@163. com。 